\title{
DETERMINATION OF TOP DEAD CENTRE LOCATION BASED ON THE MARINE DIESEL ENGINE INDICATOR DIAGRAM ANALYSIS
}

\author{
Roman VARBANETS ${ }^{1}$, Vitalii ZALOZH ${ }^{2}$, Anatoliy SHAKHOV ${ }^{1}$, Iryna SAVELIEVA ${ }^{1}$, \\ Varvara PITERSKA ${ }^{1}$ \\ ${ }^{1}$ Odessa National Maritime University, Ukraine, e-mail: roman.varbanets@gmail.com \\ ${ }^{2}$ Danube Institute of the National University “OMA”, Ukraine, e-mail: zalogh@ukr.net
}

\begin{abstract}
The article discusses methods for determining top dead center, based on the analysis of pressure diagrams of a marine engine. The advantages and disadvantages of different methods are shown. Diagnostics of marine engines during operation and the selection of optimal operating conditions is based on the analysis of gas pressure diagrams, as well as fuel supply and gas distribution diagrams. As a result of the analysis of the pressure diagrams, the indicator engine power is calculated, which is further used in the management of engine operation modes, in the calculation of specific indicators, as well as in the calculation of energy efficiency coefficients of marine vessels according to International Maritime Organization recommendations. The influence of the accuracy of determining the position of the top dead center on the calculation of the mean indicated pressure and indicator power is shown. The authors considered a method for determining top dead center, based on the solution of the equation $P^{\prime}=0$, which provides the required accuracy in calculating the mean indicated pressure and indicator power of the engine during operation. It is shown that the method can be applicable in marine engine working process monitoring systems as an alternative to hardware methods for determining the top dead center.
\end{abstract}

Keywords: diesel engine, top dead center, TDC pickup sensor, cylinder pressure monitoring, mean indicated pressure, indicated power, ship energy efficiency coefficient

\section{INTRODUCTION}

Diagnostics of marine engines and the selection of optimal operating modes are based on the analysis of indicator pressure diagrams in the working cylinder depending on the angle of crankshaft rotation $\left({ }^{\circ} \mathrm{CA}\right) P(\varphi, \mathrm{deg})[1-3]$. In parallel with indicator diagrams obtained during operation, fuel supply and gas distribution diagrams can be considered, which increase the reliability of diagnostics [4 - 6]. As a result of the analysis of indicator diagrams, the mean indicated pressure $(M I P)$ and indicator power (iPower) are calculated, which is further used in the management of engine operation modes, in the calculation of specific indicators, as well as in the calculation of energy efficiency coefficients of marine vessels according to International Maritime Organization (IMO) recommendations [7]. The error in determining the position of the top dead center (TDC) has the greatest influence on the accuracy of the indicator power calculation [4].

The indicator diagrams are recorded on the engine under maximum continuous rating (MCR) load of an engine using special pressure sensors. Such sensors ensure stability of characteristics at high temperature of the medium being measured (IMES sensors [8], Kistler sensors [9], etc.). The pressure values are recorded in the computer memory in the form of time series $P(t)$ with a constant step. In most cases, the sampling step is chosen so as to obtain at least two points per one degree of the crankshaft rotation.

The task of obtaining indicator diagrams from the time series $P(t)$ is formulated as the translation of data from functions of time $t$ as a function of the angle of the crankshaft rotation $\varphi$, where the accuracy of piston TDC determining has the greatest influence. Between the TDC of adjacent cycles, an assumption is made that the speed of rotation is constant, which allows to convert the time into angles of rotation of the crankshaft. Such a rough assumption nevertheless takes place in the problems of diagnosing the technical condition of cylinders.

$$
f(t) \stackrel{T D C}{\longrightarrow} f(\varphi)
$$

There are two methods for solving this problem: hardware - using sensors on the engine flywheel (AVL OT-sensor 428 [10], Kistler TDC sensor Type 2629B [9], etc.), and analytical - by analyzing individual sections of the cylinder gas pressure diagram in the working cylinder $P(t)$ and its derivative diagrams.

\section{HARDWARE METHOD FOR THE TDC DETERMINATOIN}

The hardware method for the TDC determination is obvious and has traditionally been used on transport engines since the very first working pro- 
cess monitoring systems were used. The TDC sensor, or TDC pickup sensor, is mounted on the engine flywheel so as to mark the TDC position of the first cylinder. TDC of the remaining cylinders are determined by the angles of the crank mechanism. The high accuracy of the TDC position is ensured by halving the $[\mathrm{m}, \mathrm{m} 1]$ section on the engine flywheel. The angles from TDC to points $\mathrm{m}$ and $\mathrm{m} 1$ are selected in the range of $6 \div 10{ }^{\circ} \mathrm{CA}$ before and after TDC, Fig. 1.

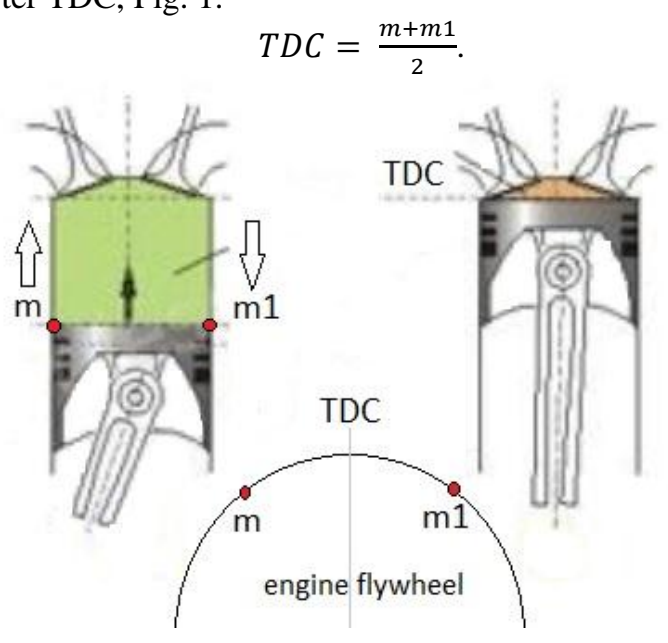

Fig. 1. Installing TDC at two points: before TDC $(\mathrm{m})$ and after TDC $(\mathrm{m} 1)$

Following systems used sensors on the flywheels for TDCs determination and monitoring systems synchronization: marine diesel monitoring systems NK-5 (one of the first widely used in the 1990s) and subsequent NK-100, NK-200 Norwegian company Autronica A/S; European systems Kyma, Premet, Malin, Doctor, Depas 2.34 [4], as well as many other systems developed before the early 2000s.

It must be pointed out that the operation of marine diesel monitoring systems using TDC hardware sensors is associated with a number of problems. First of all, it is the need for temporary decommissioning of the engine. As a rule, all work related to the need for temporary decommissioning of the marine engine should be coordinated with the maritime and coastal services, and special permission must be obtained. In addition, the installation of TDC pickup sensors requires significant time and material costs associated with the need for accurate calibration of the sensors. Additional cables connecting TDC pickup sensors, temporarily laid during engine diagnostics, reduce the reliability of the entire measurement system and increase the risk of hardware errors.

The TDC sensor is installed on an idle engine. Then, when the engine rotates under load, several events occur that shift the position of the TDC relative to its static position:

- change of micro gaps in the bearings of the crank mechanism and the piston;

- torsional vibrations of the crankshaft;
- thermodynamic displacement of TDC [11];

- displacement of the TDC when measuring pressure through the indicator valve channel [3]. These indicator valves are equipped with all the low- and medium-speed diesels from the time of receipt of a well-known Rudolf Diesel's patent No. 67207 (February 23, 1892).

All portable monitoring systems record the pressure in the working cylinder by connecting a pressure sensor to the indicator valve, which has a standard thread W27 $\times 1 / 10$. During pressure measurement, the channel introduces errors such as signal delay, throttling, and wave errors, the first one can be very significant - up to several degrees - and it increases with increasing engine speed. As a result, due to only the last factor, the TDC position can shift by several degrees relative to its static position $[3,4]$.

It became obvious that the TDC hardware sensors, which are inconvenient when working with portable systems, also introduce a number of significant errors in the calculation of the mean indicated pressure and indicator power [12, 13].

When expensive stationary monitoring systems for marine engines were replaced by portable periodic monitoring systems, analytical methods for synchronizing and calculating TDCs began to develop.

\section{THE INFLUENCE OF THE TDC DETERMINATION ERROR ON THE CALCULATION OF THE WORKING PROCESS PARAMETERS}

The error of the hardware sensors is large and variable depending on the load. It became evident that within this error, and even more precisely, the TDC can be calculated by analyzing the individual sections of recorded diagrams $\mathrm{p}(\mathrm{t})$ and diagrams derived therefrom. On the other hand, the influence of the TDC error on the accuracy of mean indicated pressure calculating is very large: $6 \div 9 \%$ per $1^{\circ}$ TDC error (M. Tazerout [12], S. Polanowski [14], Per Tunestal [15], Y. Nilsson and L. Eriksson [16], E. Pipitone [17]).

In [4], an analysis is made of the influence of the TDC determination error on the calculation of the main parameters of the working process: mean indicated pressure, indicator power, speed and degree of increase in pressure during combustion, calculated values of pressure of the fuel combustion beginning and pressure on the expansion line, Fig. 2. Due to the TDC determining error a significant distortion of the working process parameters calculation results occurs. The calculated value of the fuel injection advance angle $\alpha$ increases linearly in accordance with the synchronization error (Fig. 2a). The calculated value of the degree of increase in pressure $\lambda$ decreases linearly, and the value of the rate of increase in pressure $V_{m}$ increases and then decreases (Fig. 2b). The calculated values of the 
mean indicated pressure and indicator power of the cylinder almost linearly increase by $7.2 \% /{ }^{\circ} \mathrm{CA}$ (Fig. $2 \mathrm{c})$. The calculated value of pressure at the end of compression increases by $5.5 \% /{ }^{\circ} \mathrm{CA}$, and the control point of pressure on the expansion line $\left(36^{\circ} \mathrm{CA}\right.$ per TDC) decreases by $4.6 \% /{ }^{\circ} \mathrm{CA}$ in a linear manner (Fig. 2c).
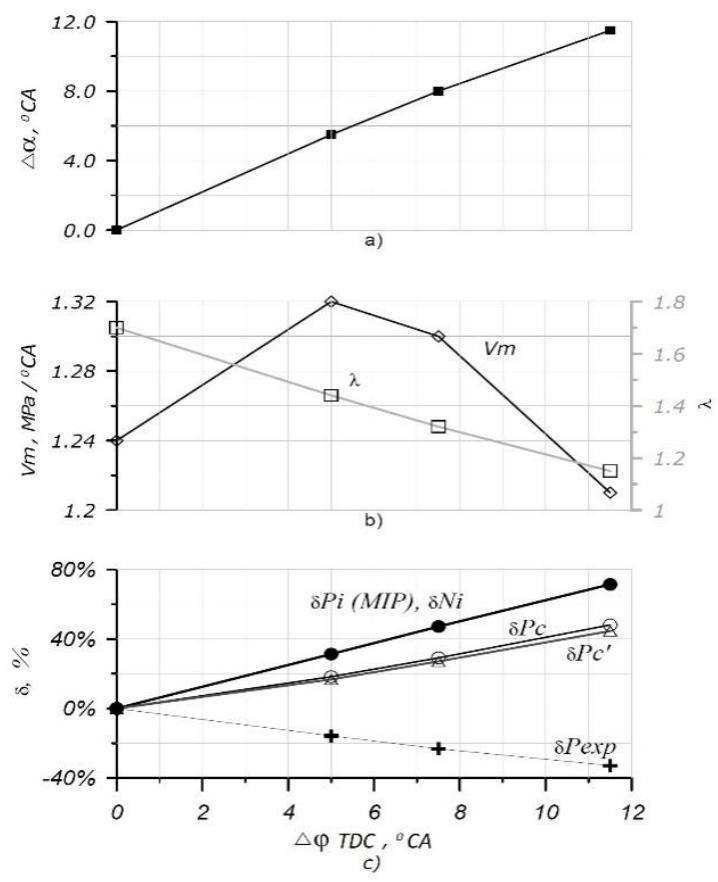

Fig. 2. The influence of the TDC determination error on the parameters of the working process of the S60MC-C main engine

Thus, in order to obtain a practical calculation of the mean indicated pressure and indicator power (with a maximum relative error of no more than $2.5 \%$ ), it is necessary to determine the TDC with a maximum absolute error of not more than $0.1 \div$ $0.25{ }^{\circ} \mathrm{CA}$.

\section{ANALYSIS OF EXISTING ANALYTICAL METHODS FOR DETERMINING TDC}

Perhaps one of the first analytical methods for determining TDC (rather, correction of TDC) used in practice, was the method implemented in the marine diesel engines diagnostic systems «The Electronic Indicator Lemag Premet XL, C» [18] (Fig. 3).

The system provides semi-automatic (involving operator) constructing the tangent to the curve of the cylinder pressure change rate before TDC (Fig. $3 a)$. The coordinate of the intersection of this tangent zero (on a scale $d P / d \varphi$ ) is assumed to be the adjusted TDC value. It is proposed to shift the entire indicator diagram (adjust the position of the TDC) so that this tangent crosses " 0 " at the TDC position (Fig. 3b). Further, the system manual says that such a method is based on "... the fundamental thermodynamic laws of the working process in the ICE cylinder". This implies the fact that in the ab- sence of fuel supply (during compressionexpansion in the cylinder without combustion), the rate of change in pressure at TDC should be zero.
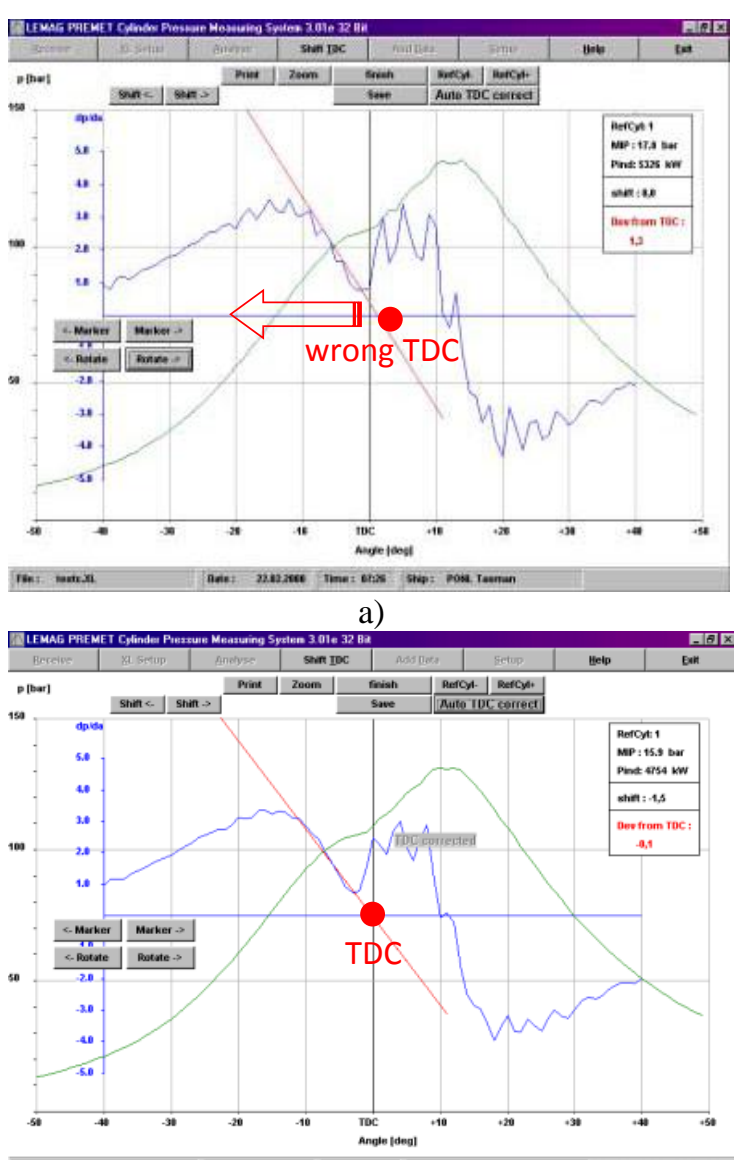

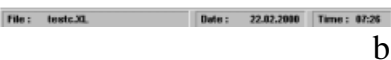

Fig. 3. TDC adjustment in LEMAG systems "PREMET XL, C" [18]

This statement is not entirely correct, because there is a thermodynamic displacement of the pressure diagram due to the transfer of heat to cylinder walls $[3,11]$. Due to thermodynamic displacement, the entire pressure diagram shifts slightly to the left, the compression maximum is up to the TDC and, accordingly, the curve $d P / d \varphi$ also crosses " 0 " slightly to the left of the actual TDC value. This fact can be taken into account due to the constant correction of TDC, because the thermodynamic displacement is very small and for most marine engines its value does not exceed $1{ }^{\circ} \mathrm{CA}$ [11]. It should be noted that the first LEMAG systems "PREMET XL, C" contained no information about this, nevertheless, the software of the systems provides for the correction of the TDC by a certain constant value.

Failure to take into account the thermodynamic displacement of real indicator pressure diagrams in the working cylinder $P(\varphi, \mathrm{deg})$ by most modern marine diesel monitoring systems can be explained by the following subjective reasons:

- the thermodynamic displacement is small (less than $1{ }^{\circ} \mathrm{CA}$ ), and its calculation can be performed only with the empirical specification of heat trans- 
fer coefficients. Calculation of these coefficients by different methods gives results that differ by almost 100\% [11-13, 15-17];

- not taking into account the thermodynamic displacement of the diagram to the left to the TDC gives the effect of an artificial increase in the mean indicated pressure and indicator power. This certainly pleases engine operators who calculate engine specific rates for regular reports.

Nevertheless, almost all modern monitoring systems have the ability to enter the constant coefficients for TDC correction. This makes it possible to take into account both the thermodynamic displacement and the systematic calculation errors.

One of the most interesting methods for correcting TDC is given by $\mathrm{M}$. Tazerout et al. [12, 20]. In these works, it was shown that the T-S diagram constructed from the measured pressure data has a near symmetrical form without intersections, in the case of the correct determination of the TDC, and the intersection with the characteristic loop, in the case of an error in TDC determination, Fig. 4.

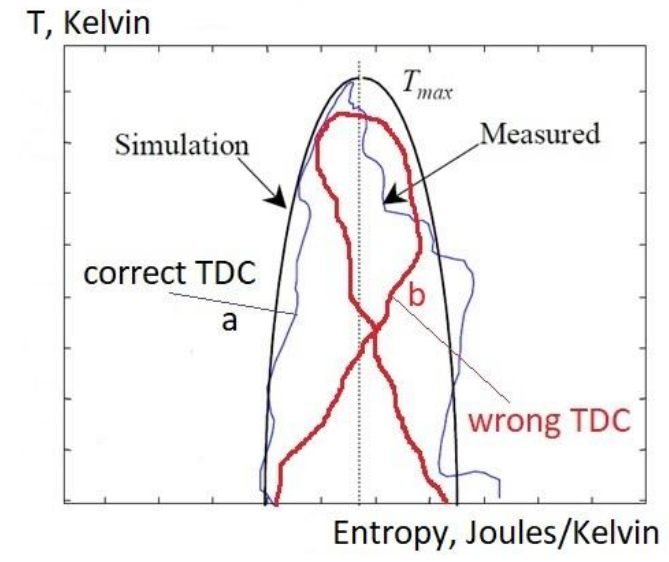

Fig. 4. T-S diagram in cases of correct (a) and erroneous (b) determination of TDC $[12,20]$

The indicated method can be implemented in practice if a strict criterion for the presence of a loop on the T-S diagram is proposed. However, the presence of noise in the data and the unstable appearance of the T-S diagram itself will certainly reduce the accuracy of this criterion.

In [22], the output of the volume in the cylinder at the point " $\mathrm{m}$ " is given - the maximum rate of pressure increases during compression, see Fig. 5. The article shows that the volume $V m$ does not depend on the pressure $P m$ and the derivative of the pressure $P^{\prime} m$, but depends only on the geometric data of the cylinder.

$$
V_{m}=V_{c}+\frac{\pi D^{2} s}{8}\left(\begin{array}{l}
1+1 / \lambda_{C R}-\cos \varphi_{m}- \\
-\Phi_{m}^{0.5}
\end{array}\right),
$$

where $\Phi_{m}=1 / \lambda_{C R}{ }^{2}-\sin ^{2} \varphi_{m}, V_{c}-$ the compression chamber volume; $\lambda_{C R}=S / 2 L_{C R}-$ the ratio of the radius of the crank to the length of the connecting rod; $D, S$-cylinder diameter and piston stroke, $L_{C R}$ - connecting rod length.

In conclusion of this article [22], it follows that the volume at the point " $\mathrm{m}$ " and, accordingly, the angle of rotation of the crankshaft from " $\mathrm{m}$ " to TDC can be calculated beforehand, before diagnosing engine, knowing the diameter of the cylinder $D$, the stroke of the piston $S$, the length of the connecting $\operatorname{rod} L_{R}$ and the volume of the compression chamber $V_{C}$. The coordinate " $\mathrm{m}$ " can be determined by numerically differentiating the diagram $P(t)$. Thus, the TDC offset in degrees ${ }^{\circ} \mathrm{CA}$ relative to the $P^{\prime} m$ point can be determined before diagnosing the engine. Such a wonderful property of the $P$ ' $m$ point could quickly solve all the problems of analytic determination of TDC and the task of analytical data synchronization when diagnosing engines. However, it must be recognized that the presence of noise in the discrete pressure data $P(t)$, even if correctly excluded [3], can significantly reduce the accuracy of this elegant method. However, this method can be effectively used in a preliminary assessment of TDC.

In [4], an analysis of the TDC determination method using the Agnesi function recorded for the compression section up to $P^{\prime} c$

$$
P(\varphi)=P C /\left[1+\theta\left(\varphi-\varphi_{T D C}\right)^{2}\right],
$$

where $P c$ and $\theta$ are the parameters determined using least squares method in the compression section by minimizing the functional

$$
\bar{F}=\sum_{j=1}^{l}\left[\frac{P_{j}}{P\left(\varphi_{j}\right)}-1\right]^{2} \rightarrow \min
$$

The main property of the Agnesi model is that the calculated TDC coordinates have a small absolute error (less than $1{ }^{\circ} \mathrm{CA}$ ) when setting the initial approximations $P c$ with an error of up to $8 \%$ [4].

In [13-17], methods based on the symmetry properties of compression-expansion curves, analysis of T-S and P-V diagrams and analysis of a thermodynamic model of a working process based on the first law of thermodynamics are presented. All these methods can be used with certain assumptions in practice if the procedure for correctly eliminating noise in the initial data $P(t)$ is performed. This procedure should not shift the phase and distort the amplitude of the original signal.

It should be noted that the method of thermodynamic modeling of the cycle requires a larger estimated time and uses empirical coefficients. It is difficult to put into practice in real time. Empirical coefficients reduce the accuracy of the calculation. The accuracy of TDC calculating of all the considered methods largely depends on the effectiveness of the noise elimination procedure in the initial data.

\section{ANALYTICAL METHODS OF THE TDC DETERMINING}

\subsection{Linear Regression Method}

The TDC method described in Section 4 used by The Electronic Indicator Lemag "Premet XL, C" system, has an important theoretical meaning, which is not fully disclosed in the system 


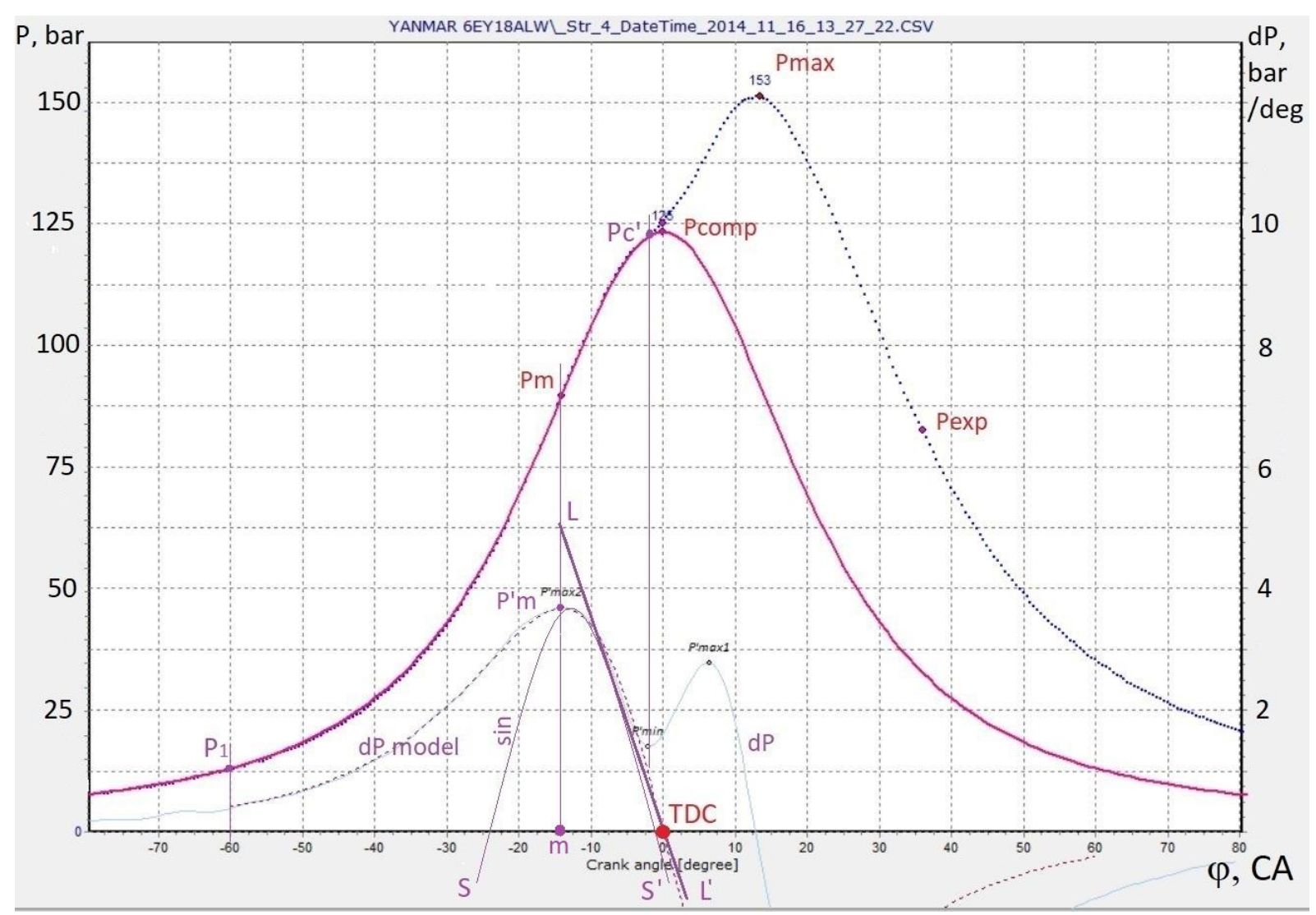

Fig. 5. Diagrams $P(\varphi, d e g), d P / d \varphi$ of a medium-speed marine diesel Yanmar 6EY18ALW

description. It can be explained if we consider the diagram $P(\varphi$, deg $)$ of a typical medium-speed marine engine and its derivative $\frac{d P}{d \varphi}$, recorded using high-quality IMES pressure sensors [2] after eliminating high-frequency noise [3], Fig. 5.

If we consider the portion of the $\frac{d P}{d \varphi}$ diagram from the maximum compression rate (point $P^{\prime} m$ ) to the start of combustion (the coordinate of point $\left.P c^{\prime}\right)$, we can see that it can be modeled by the straight line $L L$ ' or the portion of the sinusoid SS'. Despite the fact that the Electronic Indicator Lemag documentation "Premet XL, C" says that it is tangent to the inclined section of the $P$ ' chart, by all indications of further actions there is meant a linear approximation of this section. Without taking into account the thermodynamic displacement of the pressure diagram (it is negligibly small), the approximating straight line should cross " 0 " in the TDC position. This position follows from the fact that the rate of pressure change in TDC in this case is zero.

The equation of the straight line $L L^{\prime}$ can be written as

$$
L L^{\prime}=b_{0}+b_{1} \varphi .
$$

The TDC coordinate is determined from the condition

$$
L L^{\prime}=0 \rightarrow \varphi_{T D C}=-b_{0} / b_{1} .
$$

According to the least square's method requirements, we can to write the expression for $b_{1}$ and $b_{0}$ in the form

$$
b_{1}=\frac{\sum \varphi_{i} \sum P^{\prime}{ }_{i}-n \sum P_{i}^{\prime} \varphi_{i}}{\left(\sum \varphi_{i}\right)^{2}-n \sum \varphi_{i}^{2}}, b_{0}=\frac{1}{n}\left(\sum P^{\prime}{ }_{i}-b_{1} \sum \varphi_{i}\right),
$$

where the sum sign denotes the summation in the section from the maximum pressure in the compression section $\mathrm{Pm}$ to the pressure of the start of combustion in the cylinder $P c^{\prime}: \quad \sum=\sum_{i=\varphi_{i} P_{m}}^{\varphi_{i} P_{C}^{\prime}} ; n$ is the number of points on the site.

To increase the accuracy of the linear regression method, it is necessary to exclude a part of the points $\vartheta$ after the $P m$ coordinate and before the $P c^{\text {' }}$ coordinate. Thus, the basis for constructing a regression model is specified, and we can write

$$
\sum=\sum_{i=\varphi_{i} P_{m}+\vartheta}^{\varphi_{i} P_{C}^{\prime}-\vartheta} ;
$$

In the case when recording indicator diagrams implies 2 points per $1{ }^{\circ} \mathrm{CA}, \vartheta=3 \div 5$ points, depending on the type of diagram $\frac{d P}{d \varphi}$ and the actual length of the section of this diagram from $P m$ to $P c^{\prime}$.

A significant problem of the linear regression method, which significantly increases the TDC determination error, appears when the [Pm, Pc'] section of the $\frac{d P}{d \varphi}$ diagram is too small. In fig. 6 shows the indicator diagram of the MAN 9L58/64 marine engine with a large angle of fuel injection. 


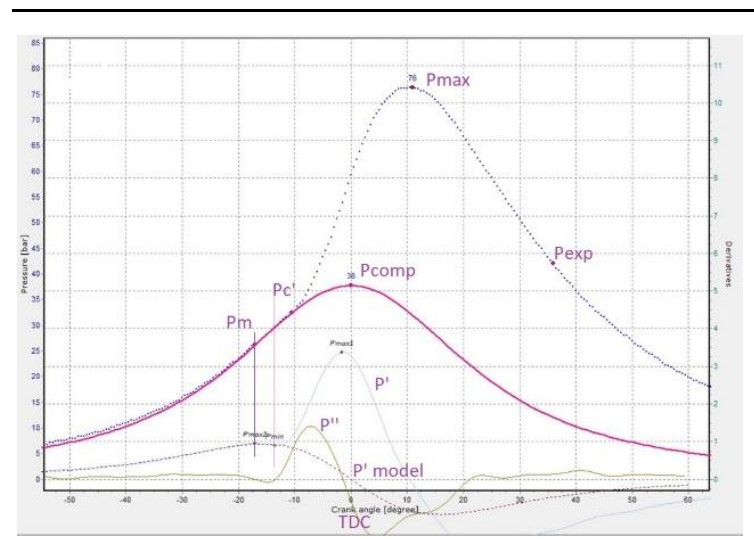

Fig. 6. Indicator diagram MAN 9L58/64 with a large angle of fuel injection timing

In the case of early angle of fuel injection timing the $\left[\mathrm{Pm}, \mathrm{Pc}^{\prime}\right]$ section of the $\frac{d P}{d \varphi}$ diagram is shortened, as shown in Fig. 6. In some cases, when the geometrical angles of fuel injection timing reach $15 \div 20{ }^{\circ} \mathrm{CA}$ to TDC, this section disappears altogether. In such cases, the TDC refinement method proposed in the Electronic Indicator Lemag "Premet XL, C" systems will work with a large error.

The most stable operation of the TDC determination method using linear regression will be on modern two-stroke marine engines with late fuel injection and the beginning of combustion later than TDC, fig. 7.

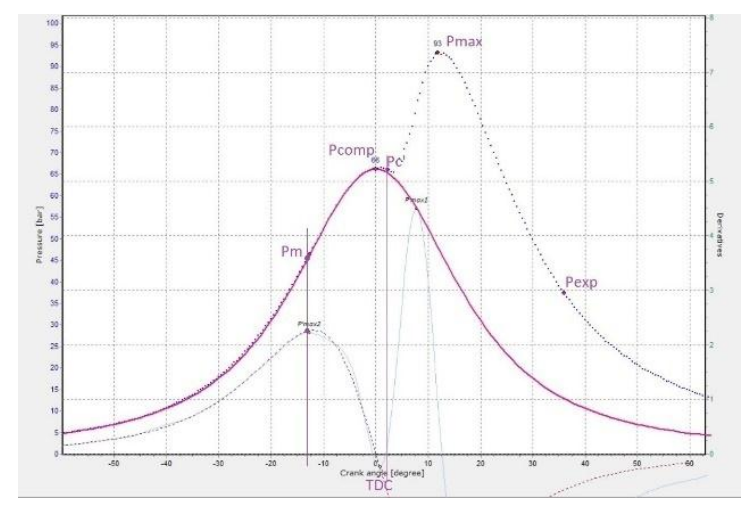

Fig. 7. Indicator diagram 6RT-FLEX82C with late injection timing

In this case, the $\left[\mathrm{Pm}, \mathrm{Pc}^{\prime}\right]$ section of the $\frac{d P}{d \varphi}$ diagram has a sufficient number of points to build a linear regression model. Moreover, the $\frac{d P}{d \varphi}$ diagram intersects " 0 ", and there is no need to extrapolate the linear model, thereby increasing the probability of error. The TDC position in this case is located inside the [Pm, Pc'] section, which makes it possible to determine the TDC with high accuracy, as, for example, for the Wartsila 6RT-FLEX82C engine (see Fig. 7).

\subsection{Sinusoidal Model}

In all cases, the derivatives of the initial diagram $P(t)$ are determined by the method of numerical differentiation, while the high-frequency noise is removed (as shown in [3]). This makes it possible to further analyze the obtained diagrams $P^{\prime}(t)$ and $P^{\prime \prime}(t)$. Using this analysis, it is possible to determine the coordinates of the extremes $P^{\prime}(t)$ and $P^{\prime \prime}(t)$ in the compression sections and, thus, determine the phase of the combustion beginning $P c^{\prime}$ ' with sufficient accuracy $[3,4]$. The $P c^{\prime}$ phase is approximately the end of the compression process in the cylinder and is the right boundary of the site simulation [Pm, Pc'].

Obviously, the linear regression model does not accurately describe the nature of the change in pressure in the $\left[\mathrm{Pm}^{\mathrm{Pc}} \mathrm{Pc}^{\prime}\right]$ section, which is not linear in its physical essence. We can even say that with a very large approximation this section is similar to a linear dependence. In addition, in the situations shown in Fig. 6, the TDC correction method proposed in the Electronic Indicator Lemag "Premet XL, C" systems will not work at all or will work poorly.

Obviously, the [Pm, Pc'] section of the $\frac{d P}{d \varphi}$ diagram can be modeled with a significantly smaller error by the sinusoidal SS' model (see Fig. 5). In this case

$$
\begin{gathered}
S S^{\prime}=P_{m}^{\prime} \sin \left(\nabla_{i}\right), \\
\text { where } \nabla_{i}=B \varphi_{i}+A,
\end{gathered}
$$

where $B, A$ are the coefficients of the sinusoidal model; $P$ ' $m$ is the maximum of the first derivative pressure diagram in the compression section.

Unlike the linear model, a part of the $\frac{d P}{d \varphi}$ curve to the $P$ ' $m$ point is used to construct it. When building a sinusoidal model, up to $\vartheta=6 \div 10$ points can be used before the Pm coordinate and after the Pc' coordinate (when recording indicator diagrams with a step of 2 points per $1^{\circ} \mathrm{CA}$ ).

The coefficients of the sinusoidal model SS' are determined by minimizing the $F_{S}$ functional constructed according to the requirements of the least squares method

$$
F_{S}=\sum\left[P_{m}^{\prime} \sin \left(\nabla_{i}\right)-P_{i}^{\prime}\right]^{2} \mapsto \min ,
$$

where the sum sign denotes the summation on the modeling section: $\sum=\sum_{i=\varphi_{i} P_{m}-\vartheta}^{\varphi_{i} P_{C}^{\prime}+\vartheta}$.

The sinusoidal SS 'model, with greater accuracy than the linear LL' model, describes the portion of the $\frac{d P}{d \varphi}$ curve before the start of combustion and allows determining the TDC coordinate with a smaller absolute error.

At the minimum point of the functional $F s$, all partial derivatives of the objective function should be equal to zero:

$$
\left\{\begin{array}{l}
\frac{\partial F s}{\partial P_{m}^{\prime}}=0 \\
\frac{\partial F}{\partial B}=0 \\
\frac{\partial F}{\partial A}=0
\end{array}\right.
$$




$$
\left\{\begin{array}{l}
\sum\left[2\left[P_{m}^{\prime} \sin \left(\nabla_{i}\right)-p_{i}{ }^{\prime}\right] \sin \left(\nabla_{i}\right)\right]=0 ; \\
\sum\left[2\left[P_{m}^{\prime} \sin \left(\nabla_{i}\right)-p_{i}{ }^{\prime}\right] P_{m}^{\prime} \cos \left(\nabla_{i}\right) \varphi_{i}\right]=0 ; \\
\sum\left[2\left[P_{m}^{\prime} \sin \left(\nabla_{i}\right)-p_{i}\right] P_{m}^{\prime} \cos \left(\nabla_{i}\right)\right]=0 .
\end{array}\right.
$$

Finally, we get a system of 3 equations

$$
\left\{\begin{array}{l}
P_{m}^{\prime}=\frac{\sum p_{i} \sin \left(\nabla_{i}\right)}{\sum \sin ^{2}\left(\nabla_{i}\right)} ; \\
\frac{\sum p_{i} \sin \left(\nabla_{i}\right)}{\sum \sin ^{2}\left(\nabla_{i}\right)}=\frac{\sum p_{i} \cos \left(\nabla_{i}\right) \varphi_{i}}{\sum \sin \left(\nabla_{i}\right) \cos \left(\nabla_{i}\right) \varphi_{i}} ; \\
\frac{\sum p_{i} \sin \left(\nabla_{i}\right)}{\sum \sin ^{2}\left(\nabla_{i}\right)}=\frac{\sum p_{i} \cos \left(\nabla_{i}\right)}{\sum \sin \left(\nabla_{i}\right) \cos \left(\nabla_{i}\right)} .
\end{array}\right.
$$

The system is solved numerically. The algorithm of the Powell'64 method [19] - a search in a system of conjugate directions - is well suited to minimize smooth functions of even degrees and for trigonometric functions. If we use this method, then the coefficients $P_{m}^{\prime}, B, A$ are determined after several successive steps to minimize the functional $\overline{F_{S}}$.

$$
\begin{gathered}
\overline{F_{S}}=\left(P_{m}^{\prime}-\frac{\sum p_{i} \sin \left(\nabla_{i}\right)}{\sum \sin ^{2}\left(\nabla_{i}\right)}\right)^{2}+ \\
+\left(\frac{\sum p_{i} \sin \left(\nabla_{i}\right)}{\sum \sin ^{2}\left(\nabla_{i}\right)}-\frac{\sum p_{i} \cos \left(\nabla_{i}\right) \varphi_{i}}{\sum \sin \left(\nabla_{i}\right) \cos \left(\nabla_{i}\right) \varphi_{i}}\right)^{2}+ \\
+\left(\frac{\sum p_{i} \sin \left(\nabla_{i}\right)}{\sum \sin ^{2}\left(\nabla_{i}\right)}-\frac{\sum p_{i} \cos \left(\nabla_{i}\right)}{\sum \sin \left(\nabla_{i}\right) \cos \left(\nabla_{i}\right)}\right)^{2} \mapsto \min .
\end{gathered}
$$

The minimum of the functional $\overline{F_{S}}$ found from the starting point $\left(P_{m}^{\prime}, 0,0\right)$ (Fig. 5) determines the TDC coordinate. In this case, the Powell'64 method determines the global minimum of the functional $\overline{F_{S}}$ in less than 10 search steps for all the studied Pdiagrams of different types of marine engines. To implement the method, it is necessary to have an initial approximation of the TDC and the angles of rotation of the crankshaft $\varphi$, which were obtained using a linear regression model.

\subsection{Model $\boldsymbol{P}^{\prime}=0$}

When constructing a linear least-square regression model, a small portion of the numerically taken derivative $\frac{d P}{d \varphi}$ is used: $\left[\varphi_{i} P m+\vartheta, \varphi_{i} P^{\prime} c-\vartheta\right]$. When building a sinusoidal model, a slightly larger section is used $\left[\varphi_{i} P m-\vartheta, \varphi_{i} P^{\prime} c-\vartheta\right]$. In those situations where the angle of injection timing is close to " 0 " or less (late injection), the base of points in these areas is sufficient to build models (Fig. 5, 7).

Nevertheless, for engines with an early injection timing (Fig. 6), these sections are too small to construct regression models.

If we use the model of the rate of change in pressure in the compression section obtained from the equation of the polytropic compression $P V^{n}$ $=$ const, then we can use a large base of experimental points. For example, we can use all the points from the beginning of the compression process (after closing the intake valves) to the start of combustion in the cylinder. However, when analyzing real indicator diagrams, the influence of noise in the areas after the valves are closed is too large, which introduces an additional error in the construction of the regression model [3]. The influence of noise is much less if we consider the portion of the diagram from the coordinate $P_{1} \sim 0.1 P \max$ (see Fig. 5) to the coordinate $P^{\prime} c$. Thus, the basis for constructing the model $P^{\prime}=0$ is the part of the diagram $\left[\varphi_{i} P_{l}, \varphi_{i} P^{\prime} c-\vartheta\right]$.

The gas pressure in the specified compression section is [1]

$$
\tilde{p}_{c o m p}=P_{a}\left(\frac{V_{a}}{V_{\varphi}}\right)^{n 1}
$$

where $P_{a}$ is the pressure at the beginning of compression;

$\boldsymbol{V} \boldsymbol{\varphi}$ is the current volume of the cylinder.

$$
\begin{aligned}
V_{\varphi}= & V_{C}+0,5 V_{S}\left[1+\frac{1}{\lambda_{C R}}-\cos \varphi-\right. \\
& \left.\frac{1}{\lambda_{C R}} \sqrt{1-\left(\lambda_{C R} \sin \varphi\right)^{2}}\right] ;
\end{aligned}
$$

$\boldsymbol{V}_{\boldsymbol{s}}=\boldsymbol{V}_{\boldsymbol{c}}(\boldsymbol{\varepsilon}-\mathbf{1})-$ the volume described by the full stroke of the piston;

$\mathbf{V}_{\mathbf{c}}$ is the volume of the compression chamber;

$\varepsilon$ is compression ratio in the cylinder;

$\lambda_{C R}=R_{C R} / L_{C R}=S / 2 L_{C R}-$ the ratio of the radius of the crank to the length of the connecting rod.

$\boldsymbol{L}_{\boldsymbol{C} \boldsymbol{R}}$ value can be measured directly on the engine.

Both $\boldsymbol{V}_{\boldsymbol{c}}$ and $\boldsymbol{\varepsilon}$ values can be obtained from the engine documentation provided by the manufacturer.

In relation to the task of TDC determining, we take the value $n l$ constant. According to the recommendations [1] for low-speed and mediumspeed engines, the value $n l$ is assumed to be $1.34 \div$ 1.37. Further analysis showed that in the problem of determining the TDC, such an assumption is appropriate and an approximate average value of $n l$ within the indicated limits does not impair the quality of the simulation $d P / d \varphi$. Modeling quality greatly depends on the level of noise on the compression line and on the accuracy of determining the coordinates of the end compression $P c^{\prime}$, - modeling right border area [3].

The expression for the rate of pressure change at the compression section can be written

$$
\frac{d P_{c o m p}}{d \varphi}=-P_{a} V_{a}^{n 1} n_{1} \frac{1}{V_{\varphi}^{n 1+1}} \cdot \frac{d V_{\varphi}}{d \varphi} .
$$

After simple transformations, we finally obtain $d P \operatorname{comp} / d \varphi=$

$$
=-P_{a} V_{a}^{n 1} n_{1} \frac{1}{V_{\varphi}^{n 1+1}} \cdot 0,5 V_{S}\left(\sin \varphi+\frac{\lambda_{C R} \sin \varphi \cos \varphi}{\sqrt{1-\lambda_{C R}^{2} \sin ^{2} \varphi}}\right) \text {. }
$$

In this case, the parameters of the $d P \operatorname{comp} / d \varphi$ model are $P a$ and $n_{l}$. According to the requirements of the least square's method, we write the $F_{P}$ functional, minimization of which will determine the values of these parameters in the section $\left[\varphi_{i} P_{l}\right.$, $\left.\varphi_{i} P^{\prime} c-\vartheta\right]$. 


$$
F p=\sum_{j=1}^{m}\left(P_{j}^{\prime}-\frac{d P_{\text {comp }}}{d \varphi_{j}}\right)^{2} \mapsto \min
$$

where $m$ is the number of points in the section $\left[\varphi_{i} P_{l}, \varphi_{i} P^{\prime} c-\vartheta\right]$.

To minimize the $F_{P}$ functional, we use the Powell-64 method, since it is effective for minimizing quadratic and trigonometric functions.

To remove noise in these areas, the authors use the Butterworth digital filter [3, 4], which has a smooth characteristic in the entire frequency range and does not shift the phase of the original signal. The results of modeling the rate of pressure change $d P \operatorname{comp} / d \varphi$ in the compression areas before the start of $P^{\prime} c$ combustion on different types of indicator diagrams are shown in fig. 5-7.

Using a relatively large base of experimental points $\left[\varphi_{i} P_{l}, \varphi_{i} P^{\prime} c-\vartheta\right]$ to build the $d P \operatorname{comp} / d \varphi$ model gives good results. The model constructed in this way describes with high accuracy the compression process for different types of engines, even in the case of significantly earlier angles of the beginning of fuel combustion in the cylinder, as in Fig. 6.

The right boundary of the $\varphi_{i} P^{\prime} c-\vartheta$ simulation has the greatest influence on the accuracy of the model construction, since after injection of fuel into the cylinder pre-flame processes occur and the pressure curve no longer has the character of a compression polytropic. The problem of determining the coordinates of the start of combustion $P^{\prime} C$ and the right boundary is solved by analyzing the extrema of the diagrams of the derivatives of the pressure diagram, as shown in $[3,4]$.

\section{CONCLUSIONS}

The TDC determination using sensors, used in stationary monitoring systems, requires additional correction due to the displacement of the TDC position mark during engine operation under load. In this case, it is necessary to take into account the change in micro-backlash in the bearings of the crank mechanism and the thermodynamic displacement of the maximum compression pressure in the cylinder [11-13]. For stationary systems, the proposed analytical method can clarify the TDC position determined using sensors [1, 2, 8-10].

In portable diagnostic systems, the TDC determination is best done immediately by the analytical method. First of all, there are inconveniences during the diagnosis of engines, because additional pickup sensors and their cables must be used. Before installing the sensors, it is necessary to take the engine out of operation. The installed sensors must be calibrated, which is associated with a significant investment of time $[6,9,10,18]$. All portable systems for parametric engine diagnostics measure the pressure in the working cylinder through the channel of the indicator valve. In this regard, in portable systems, in addition to the aforementioned errors, errors occur such as throttling and delay of the signal, which leads to an additional shift of the TDC position [1, 3]. Thus, recently, most modern portable systems for diagnosing marine diesel engines do not use pickup sensors, but use different variants of the algorithmic determination of TDC $[2-5,8]$.

Due to the fact that indicator diagrams are recorded in the computer's memory in the form of time series $P(t)$, the linear regression method is the first method for the analytical determination of TDC. For the linear regression method, the preliminary assessment of the angles of the ${ }^{\circ} \mathrm{CA}$ is not needed.

After the initial determination of the TDC using the linear regression method, a preliminary transformation $f(t) \stackrel{T D C}{\longrightarrow} f(\varphi)$ of several consecutive operating cycles is performed. As a result, the method of sinusoidal approximation of the pressure change rate $d P \operatorname{com} p / d \varphi$ can be applied, which refines the position of the TDC of several cycles.

The final position of the TDC and the translation of the diagrams from the time series to $\varphi$ diagrams $f(t) \stackrel{T D C}{\rightarrow} f(\varphi)$ is carried out by solving the $P^{\prime}=0$ equation. The initial TDC approximation of several cycles was obtained at the previous step using a sinusoidal model.

The specified algorithm allows you to calculate the position of the TDC with a maximum absolute error of not more than $0.1 \div 0.25{ }^{\circ} \mathrm{CA}$. This ensures the required accuracy of the final calculation of the mean indicated pressure with the subsequent calculation of the indicator and effective engine power with a maximum relative error of no more than $2.5 \%$.

Such accuracy in determining the engine power allows predicting its load, diagnosing, calculating specific parameters and energy efficiency coefficients in accordance with IMO requirements. This generally improves the efficiency of marine engines.

For portable systems for engine's working process monitoring the proposed method of TDC analytical determination and subsequent data synchronization provides the following advantages:

- reduces the number of sensors and cables during diagnosis, which reduces the likelihood of failures and errors;

- TDC displacements are automatically taken into account due to various factors during operation;

- the influence of the indicator valve's channel is automatically taken into account;

- the error of the TDC analytical determination method and the subsequent synchronization of indicator diagrams is much less than the hardware TDC determination method;

- no preliminary preparation of the engine is needed, and the engines can be diagnosed immediately during normal operation. 


\section{ACKNOWLEDGEMENT}

The authors thank the IMES GmbH company for providing the data sets employed in this work.

\section{REFERENCES}

1. Heywood JB. Internal combustion engine fundamentals. New York: McGraw-Hill. 1988.

2. Neumann S. High temperature pressure sensor based on thin film strain gauges on stainless steel for continuous cylinder pressure control. CIMAC Congress. Hamburg. Digest. 2001: 1-12.

3. Neumann S, Varbanets R, Kyrylash O, Yeryganov OV, Maulevych VO. Marine diesels working cycle monitoring on the base of IMES GmbH pressure sensors data. Diagnostyka. 2019;20(2):19-26. https://doi:10.29354/diag/104516.

4. Varbanets RA. Diagnostic control of the working process of marine diesel engines in operation. Dissertation of the Doctor of Technical Sciences. Odessa, 2010.

5. Varbanets R. Analyse of marine diesel engine performance. Journal of Polish CIMAC. Energetic Aspects. - Gdansk: Faculty of Ocean Engineering and Ship Technology Gdansk University of Technology. 2012; 7(1): 269-275. https://doi.org/10.33082/td.2018.2-3.09

6. Varbanets R, Karianskyi S, Rudenko S, Gritsuk I. Improvement of diagnosing methods of the diesel engine functioning under operating conditions. SAE Technical Paper 2017-01-2218, 2017.

7. Resolution MEPC.282(70). 2016 Guidelines for the development of a ship energy efficiency management plan (SEEMP). International Maritime Organization (IMO). Available from: http://www.imo.org/ en/KnowledgeCentre/IndexofIMOResolutions/Marin e-Environment-Protection-Committee(MEPC)/Documents /MEPC.282(70).pdf

8. IMES cylinder pressure sensors. Available from: https://www.imes.de

9. Pressure and TDC sensors from Kistler. Available from: https://www.kistler.com

10. AVL OT-sensor 428. Available from: https://www.avl.com

11. Ding Y, Stapersma D, Grimmelius HT. Cylinder process simulation with heat release analysis in diesel engine. Power and Energy Engineering Conference, 2009. APPEEC 2009. Asia-Pacific. https://doi.org/10.1109/APPEEC.2009.4918248

12. Tazerout $\mathrm{M}$, Le Corre $\mathrm{O}$, Rousseau S. TDC determination in IC engines based on the thermodynamic analysis of the temperature-entropy diagram. SAE Technical Paper 1999-01-1489, 1999. https://doi.org/10.4271/1999-01-1489

13. Staś M. An universally applicable thermodynamic method for T.D.C. determination. SAE Technical Paper 2000-01-0561, 2000.

https://doi.org/10.4271/2000-01-0561

14. Polanowski S. Determination of location of Top Dead Centre and compression ratio value on the basis of ship engine indicator diagram. Polish Maritime Research. 2008; 2(56): 2008. https://doi.org/10.2478/v10012-007-0065-2

15. Per Tunestal. Model based TDC offset estimation from motored cylinder pressure data. Proceedings of the 2009 IFAC Workshop on Engine and Powertrain
Control, Simulation and Modeling IFP. RueilMalmaison, France, Nov 30 - Dec 2, 2009, https://doi.org/10.3182/20091130-3-FR-4008.00032

16. Nilsson Y, Eriksson L. Determining TDC position using symmetry and other methods. SAE Technical Paper 2004-01-1458, 2004, https://doi.org/10.4271/2004-01-1458.

17. Pipitone E, Beccari A. Determination Of TDC in internal combustion engines by a newly developed thermodynamic approach. Applied Thermal Engineering, 2009. https://doi.org/10.1016/j.applthermaleng.2010.04.012

18. Lemag PreMet XL. Cylinder pressure indicator. Available from: http://www.lemag.de/

19. Powell MJD. An efficient method for finding the minimum of a function of several variables without calculating derivatives, The Computer Journal, 1864; 7(2):155-162, https://doi.org/10.1093/comjnl/7.2.155

20. Tazerout M, Le Corre O, Stouffs P. Compression ratio and TDC calibrations using temperature entropy diagram. SAE Technical Paper 1999-013509, 1999. https://doi.org/10.4271/1999-01-3509

21. Wierzbicki S, Śmieja M, Grzeszczyk R. Zintegrowane sterowanie stanowiskiem badawczym silników o ZS w środowisku fast prototyping. Combustion engines. 2013;154(3): 536-541.

22. Yeryganov O, Varbanets R. Features of the fastest pressure growth point during compression stroke. Diagnostyka. 2018; 19(2):71-76. https://doi.org/10.29354/diag/89729

Received 2019-10-18

Accepted 2020-01-14

Available online 2020-01-15

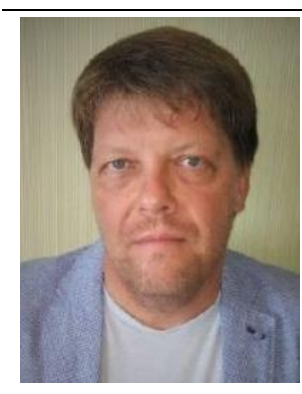

Roman VARBANETS Dr.Sc. defended his doctoral dissertation in Technical Sciences in 2010. Currently he is professor of marine engineering head of "The Marine Power Plants and Technical Operation" department in Odessa National Maritime University. Member of TAU Ukraine, IMES GmbH consultant.

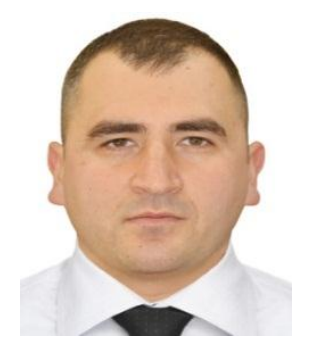

Vitalii ZALOZH is a postgraduate student at the Engineering Sciences Department in Danube institute of National university «Odessa maritime academy», Ukraine. He is a lecturer of this department.

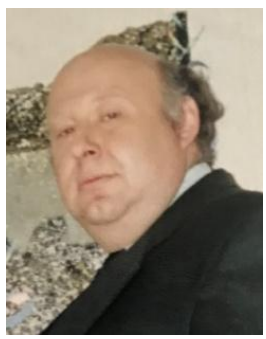

\section{Anatoliy SHAKHOV} Dr.Sc., defended his doctoral dissertation in Technical Sciences in 2007. Currently he is the professor in shipping companies' technical management area, as well as vice-rector of Odessa National Maritime University. 


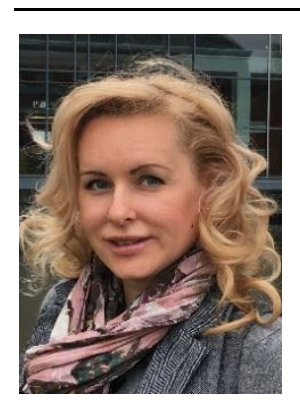

Iryna SAVELIEVA Doctor of Economics, professor of the Department of Shipping, performs research in the field of improving the efficiency of maritime transportation in Odessa National Maritime University

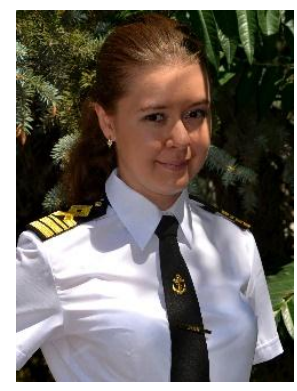

Varvara PITERSKA, Dr.Sc., defended her doctoral dissertation in Technical Sciences in 2018. Currently she is Professor at the Department of Port Operations and Cargo Works Technology in Odessa National Maritime University. Corresponding Member of Transport Academy of Ukraine. 